\title{
ON FINITENESS OF THE NUMBER OF STABLE MINIMAL HYPERSURFACES WITH A FIXED BOUNDARY
}

\author{
BY FRANK MORGAN
}

\begin{abstract}
Can there be infinitely many minimal hypersurfaces with a given boundary in a Riemannian manifold? A number of previous results, positive and negative, already indicated that the answer depends on the definition of surface, on orientability, on stability and minimizing properties of the surface, on the smoothness and geometry of the boundary, and on the ambient manifold.
\end{abstract}

1. Finiteness for area-minimizing hypersurfaces in certain manifolds. Several years ago, R. Hardt and L. Simon [HS, 1979, §12], following the original work of F. Tomi [T2, 1974], proved that a $C^{4, \alpha}$ Jordan curve $\Gamma$ in $\mathbf{R}^{3}$ bounds only finitely many orientable, area-minimizing immersed manifolds-with-boundary of least area. The immersed manifolds are assumed to minimize area over all immersed manifolds of finite topological type. It has been more or less understood that this finiteness theorem applies as well to hypersurfaces in $\mathbf{R}^{n}(n \leq 7)$, bounded by a compact, connected, $C^{4, \alpha}$ submanifold $B$ of $\mathbf{R}^{n}$.

Such finiteness for oriented area-minimizing hypersurfaces fails in general ambient manifolds. For example, in the standard 2-dimensional sphere, there is a continuum of length-minimizing paths from the south pole to the north pole. However, we prove the following finiteness theorem for a noncompact, real-analytic Riemannian manifold.

THEOREM A. Fix $n \leq 7,0<\alpha<1$. Let $N$ be an $n$-dimensional, complete, connected, noncompact, real-analytic Riemannian manifold, with sectional curvature bounded above and injectivity radius bounded away from 0 . Let $B$ be a compact, oriented $(n-2)$-dimensional, $C^{2, \alpha}$ submanifold of $N$, with a positive integer multiplicity assigned to each component. Let $\mathcal{M}$ be the space of equivalence classes (under reparameterization) of $C^{2, \alpha}$ immersions $f$ of compact, orientable, $(n-1)$-dimensional $C^{2, \alpha}$ manifolds-with-boundary $M$ into $N$, such that $f \mid \partial M$ is a covering of $B$ with the prescribed multiplicities. Let $a=\inf \{$ area $f:[f, M] \in \mathcal{M}\}$. Then $\mathcal{M}_{0}=\{[f, M] \in \mathcal{M}$ : area $f=a\}$ is finite.

When the ambient manifold $N$ is compact, it is shown that infinitely many area-minimizing hypersurfaces with a common, smooth boundary necessarily give an "open book" decomposition of $N$ (cf. [Wi, G]). It follows that if $N=\mathbf{C} P^{2}$, for example, or if $N$ is nonorientable, there are only finitely many area-minimizing hypersurfaces with a common, smooth boundary.

Received by the editors April 25, 1985.

1980 Mathematics Subject Classification. Primary 53C42; 49F22.

Key words and phrases. Minimal surface, minimal hypersurface, finiteness.

(C) 1985 American Mathematical Society $0273-0979 / 85 \$ 1.00+\$ .25$ per page 
2. Finiteness for stable, minimal hypersurfaces in $\mathbf{R}^{n}$. To what extent can the finiteness results for area-minimizing surfaces be extended to all minimal surfaces with nonnegative second variation for area? The following theorem gives the desired result for certain extremal boundaries in $\mathbf{R}^{n}(n \leq 6)$.

THEOREM B. For $n \leq 6,0<\alpha<1$, let $B$ be an $(n-2)$-dimensional, compact, $C^{2, \alpha}$ submanifold of $\mathbf{R}^{n}$, lying in the boundary of a $C^{2}$, uniformly convex set. Then $B$ bounds only finitely many orientable, immersed, minimal manifolds-with-boundary of nonnegative second variation.

In particular, for a system of $C^{2, \alpha}$ Jordan curves lying in the boundary of a $C^{2}$, uniformly convex set in $\mathbf{R}^{3}$, the theorem implies that the classical PlateauDouglas problem has only finitely many stable solutions of fixed topological type and that only finitely many topological types occur.

There had been much progress on the case of minimal immersions of the 2dimensional disc, with nonnegative second variation, bounded by an extremal $C^{4, \alpha}$ Jordan curve $\Gamma$. Earlier finiteness results were attained by $\mathrm{F}$. Tomi [T1] for $\Gamma$ "proper", by M. Beeson [B1] (see also [B2]) for $\Gamma$ real-analytic, and by M. Koiso $[\mathbf{K}]$ for embedded discs. We have learned that F.-H. Lin $[\mathbf{L}]$ has recently independently established finiteness for this case for $\Gamma C^{3, \alpha}$.

M. Anderson [A, Remark 4] remarks that a $C^{4, \alpha}$ Jordan curve $\Gamma$, whether or not extremal, bounds only finitely many embedded minimal discs of nonnegative second variation. Finiteness for the total number of minimal discs bounded by a generic, sufficiently smooth Jordan curve in $\mathbf{R}^{n}$ has been proved by R. Böhme and A. J. Tromba [BT1, BT2] and by L. P. Jorge [J].

3. Finiteness for the classical Plateau problem in certain manifolds. We also generalize the original finiteness theorem of F. Tomi [T2, Corollary 1] from $\mathbf{R}^{3}$ to more general ambients.

THEOREM C. Let $N$ be a 3-dimensional, complete, connected, noncompact, real-analytic Riemannian manifold, with sectional curvature bounded above and injectivity radius bounded away from 0 . Let $\Gamma$ be a real-analytic Jordan curve in $N$. Then there are only finitely many least-area solutions to the classical Plateau problem.

An earlier finiteness result of N. Quien [Q] applied inside sufficiently small normal charts. In contrast, our results are global.

4. Proofs of the finiteness theorems. The original finiteness theorem of F. Tomi [T2, Corollary 1] had four elements:

(1) the definition of minimal surfaces as conformal harmonic mappings of the disc,

(2) compactness properties of the space of minimal surfaces,

(3) an argument that a limit surface is part of a continuum of minimal surfaces,

(4) a contradiction of the existence of such a continuum by considering a volume functional.

Our definitions follow B. White's study [W] of minimal surfaces as equivalence classes of minimal immersions of manifolds. This approach permits 
generalizations to higher topological type and higher dimension. Moreover, the smoothness hypotheses on the boundary may be relaxed from $C^{4, \alpha}$ to $C^{2, \alpha}$.

Appropriate compactness results remain central to the proofs. However, the classical function theoretic methods must be replaced by the methods of rectifiable currents and varifolds of geometric measure theory. The proofs employ such concepts as flat chains modulo $\nu$ to deal with multiplicities and the first variation of the varifold at the boundary, as well as standard compactness and regularity results of geometric measure theory and partial differential equations.

The continuum argument (3), in the setting of B. White [W], generalizes to higher dimensions and general ambient manifolds $N$. The proof uses the analyticity of differential operators in function space, and requires $N$ to be real-analytic.

The final step (4), employing a volume functional, does not generalize to general ambient manifolds $N$. Instead, with the help of compactness and the continuum argument (3), it is shown that the set of points swept out by all limit minimal surfaces is essentially both compact and open, and hence all of $N$. The hypothesis that $N$ be noncompact now yields the desired contradiction.

REMARKS. One application of Theorem A is what seems to be the first regularity result for the area-minimizing normal currents of geometric measure theory. Also, our methods yield results on the invariance of minimizing hypersurfaces under isometries of the ambient manifold which leave the boundary invariant. In counterpoint, examples of nonfiniteness and noninvariance are provided.

The author is grateful for helpful conversations with many friends, including Mike Anderson, Tim Cochran, Bob Gulliver, Reese Harvey, and Rafe Mazzeo. This work was partially supported by a National Science Foundation grant.

\section{REFERENCES}

[A] Michael T. Anderson, Curvature estimates for minimal surfaces in 3-manifolds, preprint. [B1] Michael Beeson, Some results on finiteness in Plateau's problem. I, Math. Z. 175 (1980), 103-123.

[B2] _ Some results on finiteness in Plateau's problem. II, Math. Z. 181 (1982), 1-30.

[BT1] R. Böhme and A. J. Tromba, The index theorem for classical minimal surfaces, Ann. of Math. (2) 113 (1981), 447-499.

[BT2] , The number of solutions to the classical Plateau problem is generally finite, Bull. Amer. Math. Soc. 83 (1977), 1043-1044.

[G] L. Z. Gao, Applications of minimal surface theory to topology and Riemannian geometry, preprint.

[HS] Robert Hardt and Leon Simon, Boundary regularity and embedded solutions for the oriented Plateau problem, Ann. of Math. (2) 110 (1979), 439-486.

[J] L. P. Jorge, $C^{2}$ stability of curves with non-degenerate solution to Plateau's problem, Bol. Soc. Brasil. Mat. (to appear).

[K] Miyuki Koiso, On the finite solvability of Plateau's problem for extreme curves, Osaka J. Math. 20 (1983), 177-183.

[L] F.-H. Lin, The Gauss map of a minimal surface, in preparation. 
[Q] Norbert Quien, Über die endliche Lösbarkeit des Plateau-Problems in Riemannischen Mannigfaltigkeiten, Manuscripta Math. 39 (1982), 313-338.

[T1] Friedrich Tomi, On the finite solvability of Plateau's problem, Geometry and Topology, Lecture Notes in Math., vol. 597, Springer, New York, 1977, pp. 679-695.

[T2] _ 52 (1973), 312-318.

[Tr] A. J. Tromba, On the number of minimal surfaces spanning a wire, Mem. Amer. Math. Soc. No. 194 (1977).

[W] Brian White, The space of $m$-dimensional surfaces that are stationary for a parametric elliptic integrand, preprint.

[Wi] H. E. Winkelnkemper, Manifolds as open books, Bull. Amer. Math. Soc. 79 (1973), 45-51.

DePARTMENT OF MATHEMATICS, MASSACHUSETtS INSTitute OF TEChNOLogy, CAMBRIDGe, MASSACHUSETtS 02139 